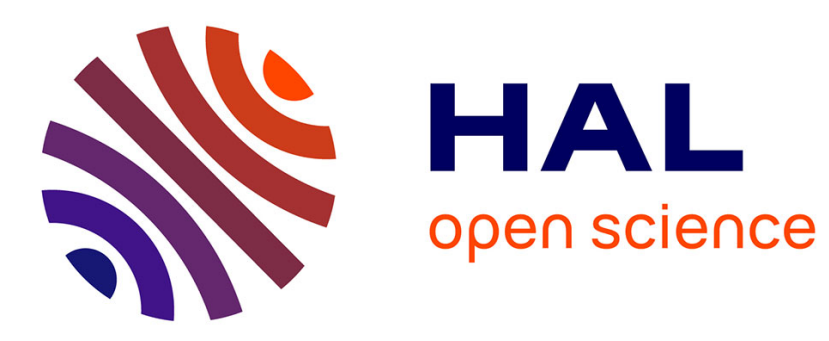

\title{
Macro-expérimentation autour des fonctions des banques centrales
}

Camille Cornand, Frank Heinemann

\section{To cite this version:}

Camille Cornand, Frank Heinemann. Macro-expérimentation autour des fonctions des banques centrales. Revue Française d'Economie, 2015, XXX (2), pp. 59-103. halshs-01232455

\section{HAL Id: halshs-01232455 \\ https://shs.hal.science/halshs-01232455}

Submitted on 20 Jun 2018

HAL is a multi-disciplinary open access archive for the deposit and dissemination of scientific research documents, whether they are published or not. The documents may come from teaching and research institutions in France or abroad, or from public or private research centers.
L'archive ouverte pluridisciplinaire $\mathbf{H A L}$, est destinée au dépôt et à la diffusion de documents scientifiques de niveau recherche, publiés ou non, émanant des établissements d'enseignement et de recherche français ou étrangers, des laboratoires publics ou privés. 


\title{
Macro-expérimentation autour des fonctions des banques centrales
}

\author{
Camille Cornand, Chargée de recherche CNRS - Laboratoire GATE* \\ Frank Heinemann, Professeur à l'Université Technique de Berlin**
}

20 mars 2015

\begin{abstract}
Résumé : L'expérimentation en macroéconomie est un domaine en pleine expansion. Cet article défend le point de vue selon lequel les expériences de laboratoire peuvent représenter un outil à la disposition des banquiers centraux et passe en revue la littérature expérimentale consacrée à l'analyse des fonctions des banques centrales. Sont ainsi abordées successivement les études relatives aux canaux par lesquels la politique monétaire affecte les comportements, aux processus de décision, et à la mise en œuvre de la politique des banques centrales. L'article conclut sur les limites et perspectives de cette approche.
\end{abstract}

Title : Macro-experiments in central banking

\begin{abstract}
Experimental macroeconomics is a fast growing field. This paper argues that laboratory experiments can represent a tool for central bankers and reviews the experimental literature on central banking issues. We successively focus on studies related to the monetary policy channels, the decision making process inside central banks, and the implementation of central banks' policy. We conclude the paper by mentioning open issues and raising new avenues for future research.
\end{abstract}

\footnotetext{
* Université de Lyon, Lyon, F-69007, France ; CNRS, GATE Lyon Saint-Etienne, Ecully, F-69130, France; e-mail: cornand@gate.cnrs.fr.

${ }^{* *}$ Technische Universität Berlin - Chair of Macroeconomics, H 52 - Straße des 17. Juni 135 - 10623 Berlin, Allemagne; email: frank.heinemann@tu-berlin.de
} 


\section{Introduction}

L'expérimentation en macroéconomie ou macro-expérimentation est un domaine en pleine expansion dans la communauté scientifique internationale, comme en témoigne la parution d'un article dans le New Palgrave sous le titre de «Experimental Macroeconomics» [Duffy, 2008b], à la suite de la rédaction par le même auteur d'une revue exhaustive de la littérature florissante sur ce thème. ${ }^{1} \mathrm{Si}$ l'échelle des phénomènes macroéconomiques est souvent perçue comme trop vaste pour être testée par des expériences de laboratoire ne comportant qu'un nombre limité d'agents - impliquant qu'il faille être conscient du paradoxe de composition et donc prudent quant à la généralisation des résultats expérimentaux dans un cadre macroéconomique agrégé -, des expériences sur des domaines ciblés de la macroéconomie peuvent permettre d'évaluer les prédictions agrégées et individuelles des modèles macroéconomiques micro-fondés. Au sein de cette littérature foisonnante, une part croissante mais encore relativement limitée de travaux s'intéresse à l'application de la macroéconomie expérimentale aux questions de politique monétaire et plus largement aux thématiques qui touchent de près ou de loin les banques centrales. Cet article a précisément pour but de présenter les expériences macroéconomiques autour des questions que se posent les banques centrales et d'évaluer dans quelle mesure ces expériences peuvent représenter des outils d'aide à la décision à la disposition des banquiers centraux. ${ }^{2}$ D'un point de vue méthodologique, cet article vise également à mieux comprendre les enjeux et les limites de l'usage de l'expérimentation pour répondre aux questions que se posent les banquiers centraux.

La macroéconomie expérimentale consiste à appliquer les outils de l'économie expérimentale à des domaines macroéconomiques. Les hypothèses et prédictions des modèles macroéconomiques ont été largement testées historiquement en utilisant des données réelles. Etant données les micro-fondations des modèles macroéconomiques, l'approche empirique alternative que représente l'expérimentation avec son environnement contrôlé et ses sujets rémunérés selon une procédure incitative se développe. En effet, l'explicitation et l'utilisation de micro-fondations dans les modèles macroéconomiques modernes comme les modèles d'équilibre général dynamiques stochastiques ("dynamic stochastic general equilibrium" (DSGE)) permettent de programmer des économies dans lesquelles les sujets prennent le rôle de divers agents économiques, mais nécessitent également le test des modules microéconomiques qui composent ces DSGE. D'après Duffy [1998, 2008a,b], tester les prédictions agrégées de modèles macroéconomiques au moyen de données réelles ne suffit pas pour discriminer entre différentes explications possibles au niveau microéconomique. Les fortes prédictions qui sont issues des théories macroéconomiques basées sur des fondements microéconomiques dépendent de la validité de ses hypothèses sur les comportements microéconomiques. Le comportement microéconomique peut être testé au moyen d'expériences de laboratoire qui fournissent un cadre permettant de contrôler les paramètres des modèles. Cette possibilité a conduit à un certain nombre d'études expérimentales testant les hypothèses des théories macroéconomiques. Un autre argument 
avancé en faveur du développement de l'expérimentation en macroéconomie tient au fait que la théorie de l'équilibre standard ne permet pas de prendre en compte l'incertitude stratégique et ne peut par conséquent donner des prédictions relatives aux mesures de politique économique, en particulier si ces modèles génèrent des équilibres multiples. Ainsi, des expériences de laboratoire peuvent être utilisées pour développer et tester les théories dans lesquelles les comportements et les anticipations dévient de manière systématique des équilibres en anticipations rationnelles. Les résultats des expériences peuvent être utiles pour répondre à des questions de sélection d'équilibre et les expériences de laboratoire peuvent être utilisées pour identifier ce qui affecte le processus de sélection parmi différents équilibres.

Pourquoi les expériences en macroéconomie sont-elles utiles pour traiter des thématiques des banques centrales ? Le recours à la macroéconomie expérimentale peut se justifier par le fait que cette dernière représente un outil permettant aux décideurs politiques de «soumettre au banc d'essai » différentes mesures concurrentes de politique. S'il est possible de faire des essais répétés de politiques alternatives spécifiques en laboratoire, il n'est pas envisageable d'effectuer un essai de politique monétaire ou de politique de communication dans la réalité. De plus, l'endogénéité de la politique dans le monde réel rend difficile l'analyse des données et la formulation d'inférences correctes sur les changements qui se sont produits. Dans le laboratoire au contraire, l'expérimentaliste détient un contrôle total sur les paramètres du modèle. L'expérimentation permet de donner une idée des différents effets - anticipés et non anticipés - de politiques alternatives. Les expériences offrent une façon rapide et à moindre coût d'identifier les conséquences d'un choix de politique monétaire. ${ }^{3}$ Comprendre la façon dont les agents économiques interagissent avec les règles institutionnelles avant la mise en œuvre de la politique permettrait de réduire considérablement le coût d'implémentation de ces politiques. ${ }^{4}$ Les expériences de laboratoire peuvent aider les responsables politiques à prendre des décisions, dans la mesure où elles permettent d'explorer les effets de mesures de politique économique alternatives et d'approximer leurs effets possibles. A l'instar d'Alan Blinder ${ }^{5}$, certains praticiens des banques centrales sont également expérimentalistes.

L'article repose sur la présentation d'expériences dont la plupart se concentrent sur le test d'hypothèses spécifiques des modèles macroéconomiques standards ${ }^{6}$; il est structuré de la façon suivante. La première section aborde les expériences relatives aux différents canaux par lesquels la politique monétaire affecte les comportements et notamment celui des anticipations. La deuxième section traite des questions de prise de décisions en matière de politique monétaire, en considérant d'une part les règles de politique monétaire mais aussi la prise de décision au sein des groupes que sont les comités de politique monétaire. La troisième section s'intéresse aux questions de mise en œuvre de la politique monétaire, avec en particulier la thématique de la transparence et des stratégies de communication des banques centrales et la question des appels d'offre en matière de refinancement. 
Enfin, la dernière section conclut sur les limites et perspectives de l'application de la macroexpérimentation à la thématique des banques centrales.

\section{Canaux de politique monétaire et anticipations}

On envisage tout d'abord la question des canaux par lesquels la politique monétaire affecte les comportements et notamment celui des anticipations.

Un certain nombre de travaux étudient les canaux de non-neutralité monétaire en laboratoire: l'illusion monétaire, l'hypothèse de rigidité de prix, et l'hypothèse de rigidité informationnelle. Pour rendre compte de cette non-neutralité au niveau agrégé, il convient également de s'intéresser à la formation des anticipations. L'importance de la gestion des anticipations pour la conduite de la politique monétaire a été soulignée notamment par Woodford [2003]. Les expériences de laboratoire génèrent des environnements contrôlés permettant d'observer comment les sujets forment leurs anticipations. Etudier la façon dont les sujets se comportent et apprennent en laboratoire peut aider à mieux modéliser les frictions internes qui conduisent à un mécanisme de propagation pertinent. En effet, les modèles en anticipations rationnelles avec rigidités nominales rencontrent des difficultés pour rendre compte des phénomènes de persistance du produit et de l'inflation. Une partie importante de la littérature relative aux phénomènes d'apprentissage, souligne que l'hypothèse de rationalité est trop forte et ne permet pas de mettre en évidence les phénomènes de persistance ; au contraire, le relâchement de l'hypothèse d'anticipation rationnelle dans des modèles économiques simples permet de mettre en évidence des mécanismes de propagation pertinents (voir par exemple Evans et Honkapohja [1993, 2001] ; Sargent [1999] ; Adam [2005]). ${ }^{7}$ Recourir à l'expérimentation pour étudier la formation des anticipations se justifie par le fait que les anticipations ne sont généralement pas facilement observables sur données réelles, ce qui ne permet pas d'identifier les déviations par rapport aux anticipations rationnelles. Au contraire, en laboratoire, les anticipations des sujets peuvent être directement observées.

\section{Illusion monétaire}

Fehr et Tyran [2001, 2005, 2008] étudient une source de non-neutralité monétaire, qui n'est généralement pas considérée dans la littérature de type DSGE : ils mettent en évidence l'illusion monétaire en laboratoire. ${ }^{8}$ Fehr et Tyran évaluent plus précisément l'impact de changements dans le niveau des prix sur la fixation des prix par les individus dans des environnements caractérisés par la présence de compléments (pour une définition et des applications du concept, voir l'encadré) et de substituts stratégiques entre leurs actions. Leur cadre expérimental envisage des sujets jouant le rôle de firmes qui fixent leur prix en terme nominal dans un marché oligopolistique. Pour les aider dans la fixation de leur prix, ils ont à leur disposition un tableau leur indiquant leurs gains en fonction de la fixation de leurs prix par les autres participants. Un changement de tableau de gains représente un gros choc anticipé sur le niveau des prix. Ils montrent que les prix répondent graduellement au choc et 
convergent vers le nouvel équilibre. La convergence est plus rapide dans les environnements où les actions des participants sont des substituts stratégiques que dans les environnements où les actions sont des compléments stratégiques.

\section{Encadré - Complémentarités stratégiques : définition, applications et résultats expérimentaux}

Définition: Un jeu présente des complémentarités stratégiques si les stratégies des joueurs peuvent être ordonnées, de sorte que des stratégies plus hautes de n'importe quel(s) joueur(s) accroissent les incitations des autres joueurs à jouer également des stratégies plus hautes. En d'autres termes, les fonctions de meilleure réponse sont croissantes. Si l'on considère un jeu symétrique à deux joueurs, dans lequel chaque joueur choisit entre une stratégie $b$ qui correspond à une action basse et une stratégie $h$ qui correspond à une action haute, et que la variation de gain d'un des deux joueurs lorsqu'il passe de la stratégie $b$ à la stratégie $h$ est négative si l'autre joueur choisit $b$ et positive s'il choisit $h$, alors les actions des deux joueurs sont des compléments stratégiques. En d'autres termes, le gain marginal de l'action haute d'un des deux joueurs augmente avec l'action haute de l'autre joueur: une action haute d'un joueur tend à induire une action haute de l'autre (pour davantage de détails, voir Cooper et John [1988] pour une présentation de la thématique des défauts de coordination en macroéconomie).

Applications en macroéconomie: De nombreux contextes macroéconomiques présentent des complémentarités stratégiques. En concurrence imparfaite, les entreprises sont amenées à ajuster leurs prix en intégrant le comportement des firmes concurrentes en matière de fixation des prix : si une firme donnée augmente ses propres prix alors que les autres ne le font pas, elle risque de subir des pertes de parts de marché. Au contraire, si les autres entreprises augmentent leur prix, le profit marginal lié à l'augmentation de son prix s'accroît pour une entreprise donnée, qui va dès lors avoir une incitation à monter son prix également. De même, sur le marché des changes, les spéculateurs attaquent une monnaie si elle est surévaluée et s'ils anticipent une attaque de la part des autres spéculateurs. Si un spéculateur attaque alors que les autres ne le font pas, le risque de subir des pertes est élevé alors que son gain peut croître avec le nombre d'agents qui décident d'attaquer. D'autres exemples liant macroéconomie monétaire et financière et compléments stratégiques peuvent être mentionnés comme les paniques bancaires, les bulles/ krachs sur les marchés boursiers, les comportements d'investissement, etc. Dans ces exemples, on observe des complémentarités stratégiques, au sens où il est d'autant plus payant de choisir telle action (par exemple attaquer une monnaie) que le nombre d'autres agents choisissant cette même action est grand.

Résultats expérimentaux : Les expériences sur les jeux de coordination montrent que les sujets convergent généralement plus lentement vers l'équilibre dans des environnements présentant des complémentarités stratégiques (voir par exemple la revue de littérature proposée par Assenza et al. [2014]). L'explication est liée à la formation des croyances et des anticipations en laboratoire. Dans ces jeux, les sujets ont des incitations relativement fortes à coordonner leurs actions. Du fait de ces profils d'actions coordonnées, il est difficile pour les sujets de détecter que leurs stratégies ne sont pas coordonnées sur l'équilibre (car les gains plus élevés associés à des déviations unilatérales sont relativement faibles lorsque les complémentarités stratégiques sont fortes). Dans les expériences de laboratoire, les sujets forment généralement des anticipations selon un processus itératif de raisonnement qui conduit à l'équilibre seulement après un nombre infini d'itérations. Dans la mesure où les sujets ne parviennent généralement à atteindre qu'un faible nombre d'itérations, ils ne parviennent pas à atteindre l'équilibre, à moins que le jeu ne soit répété un très grand nombre de fois. Ces degrés limités de raisonnement peuvent être modélisés comme des degrés limités de croyances d'ordre supérieur (les croyances d'ordre supérieur sont les croyances des agents économiques à propos des croyances des autres agents, les croyances des agents à propos des croyances des autres agents concernant les croyances des autres agents, et ainsi de suite). Les sujets échouent dès lors à converger vers l'équilibre parce qu'ils peuvent douter des croyances et du comportement des autres (voir Nagel [1995]). Ceci est source de frictions et peut expliquer la faible convergence observée vers l'équilibre.

Fehr et Tyran [2001] considèrent quatre traitements. Dans le traitement NH, les tableaux de gains sont donnés en termes nominaux et les sujets jouent avec d'autres sujets. Les gains sont ensuite transformés en monnaie réelle avec différents taux de change selon qu'ils sont réalisés avant ou après le choc. Dans le traitement NC, les autres firmes sont jouées par un ordinateur et l'unique sujet participant est informé que l'ordinateur choisit toujours un prix qui est la meilleure réponse à son propre prix. Ce traitement a pour avantage de permettre à Fehr et Tyran d'éliminer l'incertitude stratégique comme explication potentielle du délai d'ajustement des prix. Les deux autres traitements, RC et RH sont 
respectivement identiques aux traitements $\mathrm{NH}$ et $\mathrm{NC}$, mis à part que les tableaux de gains sont exprimés en termes réels et non en termes nominaux, de sorte que dans ces deux derniers traitements, l'illusion monétaire ne peut pas expliquer un éventuel délai dans l'ajustement des prix.

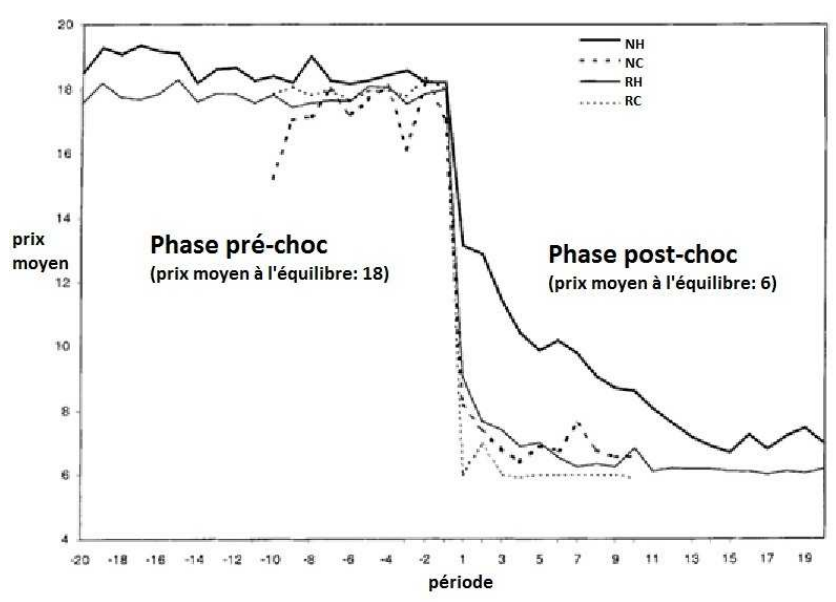

Figure 1: Prix moyen avant et après le choc pour les quatre traitements - Source : Fehr et Tyran [2001].

La Figure 1 présente le prix moyen avant et après le choc pour les quatre traitements considérés $(\mathrm{NH}$, NC, RH, RC). Dans le traitement RC, on observe un ajustement instantané sur le nouvel équilibre. Dans les traitements NC et RH, l'ajustement sur le nouvel équilibre a pris quelques périodes, mais les sujets sont parvenus à en être très proches. Dans le traitement NH (où il existe un problème de coordination et un tableau présentant des gains en termes nominaux), on observe un délai d'ajustement des prix considérable. Les déviations par rapport à l'équilibre dans le traitement NH sont plus importantes que la somme des déviations dans les traitements $\mathrm{NC}$ et RH.

Fehr et Tyran expliquent ces observations par les anticipations des sujets. Ils considèrent également que les défauts de coordination et l'illusion monétaire peuvent se renforcer mutuellement dans des environnements avec complémentarités stratégiques. L'explication fournie par Fehr et Tyran repose sur l'illusion monétaire des participants et sur l'anticipation par les sujets que les autres ajustent insuffisamment leur prix.

Fehr et Tyran [2008] font une expérience proche de la précédente, mais dans laquelle les traitements diffèrent selon que les prix sont des compléments ou des substituts stratégiques. Dans le traitement où les prix sont des substituts stratégiques, le prix moyen saute presque instantanément sur le nouvel équilibre après le choc. Au contraire, les pertes d'efficience sont beaucoup plus fortes dans le traitement où les prix sont des compléments stratégiques : les prix s'ajustent beaucoup plus lentement vers le nouvel équilibre. Les causes de cet ajustement insuffisant des prix sont à trouver dans l'illusion monétaire et les effets d'ancrage, et l'anticipation que les autres sujets souffrent d'une certaine forme de rationalité limitée.

Petersen et Winn [2014] prétendent que les résultats de Fehr et Tyran [2001] ne sont pas tant dus à l'illusion monétaire qu'à une tâche cognitive plus exigeante dans le traitement NH. Fehr et Tyran 
[2014] répondent en précisant que l'illusion monétaire ne peut apparaître que lorsque l'ajustement sur un nouvel équilibre est une tâche non triviale. Ainsi, l'illusion monétaire ne doit pas être opposée aux limites dans les capacités cognitives des individus, mais au contraire, en dépend.

\section{Concurrence monopolistique, prix rigides, information rigide}

Les délais dans l'ajustement des prix suite à un choc sont au cœur des fondements de la nouvelle macroéconomie keynésienne. Pour justifier cet ajustement, les économistes ont recours à plusieurs hypothèses théoriques: notamment (i) l'hypothèse de prix rigides [Calvo, 1983] selon laquelle les firmes ne peuvent ajuster leur prix à chaque période; (ii) l'hypothèse d'information rigide [Mankiw et Reis, 2002], selon laquelle les firmes ne peuvent mettre à jour leur information à chaque période. Des expériences ont été menées sur ces hypothèses et sur la vitesse d'ajustement des prix suite à un choc. ${ }^{9}$

Davis et Korenok [2011] présentent une expérience visant à tester la capacité relative de théories alternatives à rendre compte des délais d'ajustement des prix à la suite d'un choc nominal. Les sujets jouent le rôle de firmes qui fixent leur prix dans un cadre de concurrence monopolistique. Ils considèrent des marchés de six vendeurs et 80 périodes d'échange au cours desquelles un choc nominal doublant l'offre de monnaie se produit. Ils envisagent trois traitements. Dans le traitement BASE, les sujets jouant le rôle de firmes peuvent ajuster leur prix à chaque période et peuvent immédiatement identifier la période dans laquelle le choc se produit. Dans le traitement SP, seuls 2 des 6 sujets du marché peuvent ajuster leur prix à chaque période à tour de rôle. Dans le traitement SI, seuls 2 des 6 sujets observent le choc, de sorte que chaque firme ne reçoit l'information que toutes les 3 périodes. Les prédictions théoriques sont les suivantes. Lorsque les prix et l'information sont flexibles, on devrait observer un saut immédiat vers le nouvel équilibre dans la période suivant l'occurrence du choc. Au contraire, en information rigide et en prix rigides, on devrait observer un délai d'ajustement.

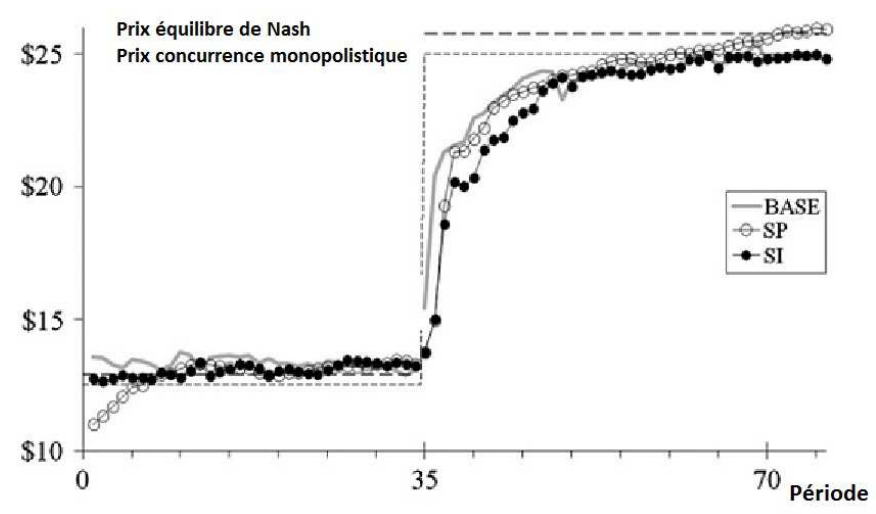

Figure 2: Prix moyens de transaction pour les traitements BASE, SP et SI, prix donné par l'équilibre de Nash et prix de concurrence monopolistique - Source : Davis et Korenok [2011].

La Figure 2 montre que même dans une économie sans friction, les sujets se comportent comme s'il y avait des frictions. En ligne avec la théorie, les déviations observées dans les traitements SP et SI 
excèdent celles observées dans le traitement BASE dans les premières et deuxièmes périodes suivant l'occurrence du choc. Dans le traitement SI, les prix s'ajustent plus lentement. Le point important est que dans les trois traitements les prix observés dévient de leurs valeurs théoriques respectives pour au moins 9 périodes sans différence significative entre les traitements pour la plupart de ces périodes. Un tel résultat peut s'interpréter de la manière suivante : même si les agents économiques peuvent obtenir de l'information et ajuster leur prix à tout moment, ils se comportent néanmoins comme si des frictions de type prix rigides ou information rigide existaient. En ce sens, les modèles théoriques proposés prix rigides et information rigide - rendent bien compte de l'observation et sont à même de capter les comportements des sujets.

\section{Anticipations et biais inflationniste}

La question du biais inflationniste est une thématique dorénavant standard dans la littérature relative à la politique monétaire. La courbe de Phillips établit le lien entre anticipations et décisions prises par le décideur politique et les agents économiques. Ce sont Barro et Gordon [1983] qui ont formalisé le biais inflationniste de la banque centrale : lorsque la banque centrale souhaite faire croître le produit au-delà de son niveau naturel, une politique monétaire discrétionnaire incite la banque centrale à tricher vis-à-vis du secteur privé en créant de l'inflation, ce qui engendre un biais inflationniste persistant. ${ }^{10}$ Plusieurs mécanismes théoriques bien connus ont été proposés pour limiter le biais inflationniste des banques centrales, notamment l'engagement (commitment) sur une règle, la crédibilité et la réputation (associées à un faible niveau d'inflation dans un jeu répété) et l'observabilité des actions de la banque centrale par les agents privés (par exemple par sa politique de communication).

En faisant jouer le rôle d'un banquier central à des sujets, les expériences de laboratoire permettent précisément d'observer l'existence d'un biais inflationniste et de tester si les mécanismes proposés permettent effectivement de réduire ce biais. Ainsi, Arifovic et Sargent [2003] et Duffy et Heinemann [2013] évaluent expérimentalement si les sujets jouant le rôle de banquiers centraux parviennent à établir la crédibilité dans le jeu de Barro-Gordon. ${ }^{11}$ Les sujets sont séparés en groupes de 4 à 6 participants ; un sujet dans chaque groupe joue le rôle d'un banquier central tandis que les autres forment des anticipations d'inflation. ${ }^{12}$ Tandis que l'expérience contextualisée d'Arifovic et Sargent $[2003]^{13}$ montre que les sujets prévoient bien l'inflation réalisée et que les autorités monétaires sont capables de manipuler leurs anticipations et de les coordonner sur l'équilibre optimal, Duffy et Heinemann [2013] présentent une version complètement décontextualisée du modèle de Barro-Gordon en laboratoire: les sujets sont informés que le joueur A (interprété par Duffy et Heinemann comme étant le banquier central) a pour tâche d'équilibrer le niveau de l'eau entre deux containers (l'un qu'ils interprètent comme le chômage, l'autre comme l'inflation); le fait de transférer l'eau d'un container à l'autre est interprété comme l'arbitrage résultant de la courbe de Phillips. Les traitements considérés sont le régime d'engagement vs. le régime discrétionnaire, ce dernier étant décliné en différents sous- 
traitements : avec et sans signaux gratuits (cheap talk), avec et sans transparence politique, avec et sans transparence économique ${ }^{14}$. Ils testent les effets des différents traitements sur les niveaux d'inflation et de chômage, sur la capacité du sujet jouant le rôle du banquier central à stabiliser l'emploi et sur le niveau de bien-être en résultant (mesuré par la fonction de paiement du sujet banquier central). Plus précisément, ils évaluent si la présence de signaux gratuits ou si la transparence peut compenser l'absence de confiance dans un jeu discrétionnaire répété. Dans le traitement avec engagement, dans lequel le sujet banquier central joue en premier et les participants au marché formant leurs anticipations connaissent l'offre de monnaie, ils montrent qu'il n'y a pas eu de biais inflationniste. Dans le traitement discrétionnaire, dans lequel le sujet banquier central décidait de l'offre de monnaie une fois les anticipations des autres sujets fixées, ils mettent au contraire en évidence l'existence d'un biais inflationniste proche de la prédiction de l'équilibre du jeu à une période. Ni l'envoi de signaux gratuits ni la transparence ne peuvent représenter des substituts à l'engagement. En conclusion, le biais inflationniste peut être mis en évidence expérimentalement. Seul l'engagement et dans une certaine mesure les effets de réputation permettent de le limiter.

\section{Formation des anticipations hétérogènes en laboratoire}

La littérature relative aux learning to forecast experiments ${ }^{15}$ (voir par exemple Bao et al. [2012]) s'intéresse à la formation des anticipations et à l'apprentissage en laboratoire. A la différence de Adam $[2007 \mathrm{a}]^{16}$, Fehr et Tyran, et de la littérature relative au biais inflationniste, les anticipations ne sont pas considérées comme homogènes parmi les agents, mais hétérogènes. Dans ce type d'expériences, les sujets ont pour tâche de prévoir le prix de certains biens pour un certain nombre de périodes, le prix de marché réalisé à chaque période étant déterminé par la moyenne des anticipations individuelles : les gains des sujets sont d'autant plus élevés que les erreurs de prévision sont faibles. Au début de chaque période, les prévisions individuelles sont collectées et réinsérées dans les fonctions de demande et d'offre. Les sujets n'ont que de l'information qualitative concernant l'économie fictive. Les courbes de demande et d'offre sont dérivées de la maximisation de l'utilité espérée et sont cohérentes avec le comportement d'optimisation rationnel. Concernant notre propos, un nombre relativement peu important d'expériences étudient dans quelle mesure les règles de politique monétaire affectent la formation des anticipations, certaines au moyen du modèle Nouveau Keynésien (NK), d'autres au sein de modèles à générations imbriquées (OLG), d'autres encore dans des DSGE.

Anufriev et al. [2013] utilisent un modèle macroéconomique stylisé de type NK pour analyser les conséquences dynamiques de l'utilisation de règles de taux d'intérêt alternatives lorsque les agents forment des anticipations hétérogènes et mettent à jour leurs croyances dans le temps. Ils montrent qu'une règle de taux d'intérêt qui vérifie le principe de Taylor ne conduit pas nécessairement le système à converger vers l'équilibre en anticipations rationnelles ; au contraire, des équilibres multiples peuvent persister. 
Pfajfar et Žakelj [2014] conduisent également des expériences de laboratoire dans un cadre NK pour explorer la formation des anticipations d'inflation. Ils analysent son interaction avec la conduite de la politique monétaire. Plus précisément, ils étudient comment la politique monétaire devrait être mise en place dans un environnement caractérisé par des anticipations hétérogènes. La version considérée du modèle NK est la suivante. La courbe IS est donnée par $y_{t}=-\varphi\left(i_{t}-E_{t} \pi_{t+1}\right)+y_{t-1}+g_{t}$, où $i_{t}$ représente le taux d'intérêt, $\pi_{t}$ l'inflation, $E_{t} \pi_{t+1}$ l'anticipation faite à la période $t$ pour la période $t+1$, $y_{t}$ l'écart de production, $g_{t}$ un choc exogène, et $\varphi$ l'élasticité de substitution de la demande intertemporelle. La courbe de Phillips est décrite par $\pi_{t}=\beta E_{t} \pi_{t+1}+\lambda y_{t}+u_{t}$.

Pfajfar et Žakelj analysent l'efficacité de différentes règles de politique monétaire à stabiliser l'inflation dans une économie où les anticipations des agents sont hétérogènes. Ils considèrent quatre traitements distincts selon la règle de politique monétaire considérée. Ils envisagent trois traitements dans lesquels la règle de taux d'intérêt (Inflation forecast targeting) est décrite par $i_{t}=\gamma\left(E_{t} \pi_{t+1}-\bar{\pi}\right)+\bar{\pi}$, de sorte que le taux d'intérêt est fixé en réponse aux déviations des anticipations d'inflation par rapport à la cible $\bar{\pi}$. Les auteurs font varier le niveau $\gamma$ d'agressivité avec lequel la banque centrale stabilise l'inflation dans chacun des trois premiers traitements $(\gamma=1.5$ dans le Traitement $1, \gamma=1.35$ dans le Traitement 2, $\gamma=4$ dans le Traitement 3 ). Le quatrième traitement ( $\gamma$ $=1.5$ ) implémente une cible d'inflation contemporaine, $i_{t}=\gamma\left(\pi_{t}-\bar{\pi}\right)+\bar{\pi}$.

L'expérience consiste à simuler des économies décrites par les trois équations précédentes et composées de 9 sujets. A chaque période $t$, les participants reçoivent un tableau leur indiquant les réalisations passées de l'inflation, de l'écart de production et du taux d'intérêt, sauf pour la première période, pour laquelle 10 valeurs initiales ont été générées par l'ordinateur sous l'hypothèse d'anticipations rationnelles. Les instructions donnaient aux participants une description qualitative de l'économie considérée. Leur tâche consistait à donner leur prévision d'inflation pour la période $t+1$, ainsi qu'un intervalle de confiance à $95 \%$ autour de leur prévision. 

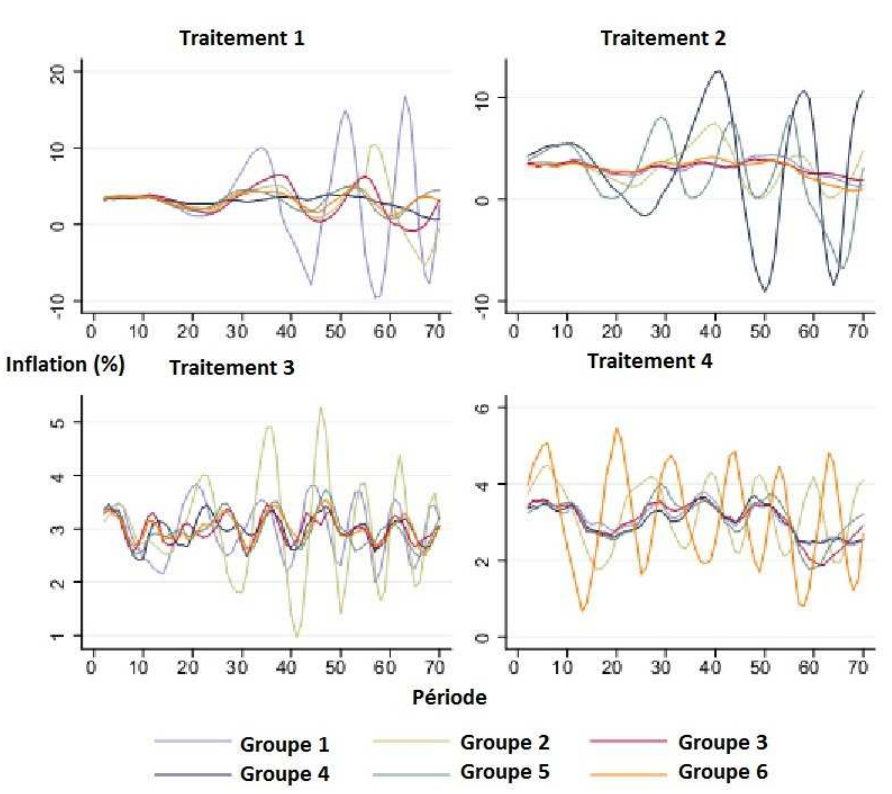

Figure 3 - Comparaison de l'inflation anticipée (prédiction moyenne des sujets par groupe) et de l'inflation réalisée par traitement - Source : Pfajfar et Žakelj [2014].

La Figure 3 donne un bon aperçu des résultats. Un plus fort degré d'agressivité de la politique monétaire (paramètre $\gamma$ ) réduit la variabilité de l'inflation, mais conduit à davantage de cycles. Une règle de ciblage d'inflation contemporaine donne de meilleurs résultats qu'une règle de ciblage des anticipations pour un même degré d'agressivité de la politique monétaire. Les auteurs complètent les résultats par une analyse de la façon dont les sujets forment leurs anticipations. Ils montrent que la plupart des sujets suivent soit un modèle d'extrapolation de la tendance, soit un modèle adaptatif (les anticipations d'inflation étant une fonction linéaire des trois variables macroéconomiques de la période précédente).

Amano, Engle-Warnick et Shukayev [2011] montrent comment les sujets forment leurs anticipations lorsque la banque centrale passe du ciblage d'inflation au ciblage du niveau des prix. Ils envisagent deux scenarios : l'un dans lequel la banque centrale cible un taux d'inflation zéro et l'autre dans lequel elle cible un niveau des prix constant. Durant l'expérience, chaque participant observait deux variables : l'inflation et le niveau des prix agrégés. Ils devaient prédire l'inflation annualisée pour la période suivante. Leurs gains dépendaient de la distance entre la valeur choisie de l'intervalle et la valeur effectivement réalisée de l'intervalle. Les auteurs montrent que les anticipations d'inflation changent selon que l'on considère un ciblage d'inflation ou un ciblage du niveau des prix : les sujets s'appuient uniquement sur l'inflation pour prévoir l'inflation future en régime de ciblage d'inflation tandis qu'ils utilisent la direction du niveau des prix dans le régime de ciblage du niveau des prix.

Marimon et Sunder [1995] et Bernasconi et Kirchkamp [2000] s'intéressent à la formation des anticipations d'inflation dans un cadre à générations imbriquées et montrent que la plupart des sujets se comportent de manière adaptative. Ces études se concentrent sur les effets de différentes politiques monétaires sur la volatilité de l'inflation. Marimon et Sunder [1995] comparent différentes règles 
monétaires et analysent leur influence sur la stabilité des anticipations d'inflation. Marimon et Sunder [1993] ont établi la relation entre stabilité et instabilité en apprentissage des équilibres en anticipations rationnelles et ont observé en laboratoire des épisodes hyper-inflationnistes.

Pour conclure, parmi les canaux de politique monétaire testés dans le laboratoire, l'illusion monétaire se démarque car elle semble liée à des effets d'ancrage sur des valeurs numériques et contraste par conséquent avec la rationalité. Nous avons vu en effet que l'illusion monétaire est liée au rôle informationnel des prix nominaux. Mais nous avons vu également qu'il est difficile de la dissocier d'autres facteurs - comme l'existence de complémentarités stratégiques - qui empêchent l'ajustement des prix après l'occurrence de chocs nominaux : la rigidité des prix peut aussi être expliquée par le fait que les agents anticipent que les autres sont affectés par l'illusion monétaire. Les expériences présentées qui introduisent explicitement des prix rigides et des informations rigides - comparant les comportements obtenus dans une économie présentant explicitement ces rigidités aux comportements observés lorsque l'économie ne présente aucune friction - montrent que même dans une économie sans friction les sujets ayant participé aux expériences se sont comportés comme s'il y avait eu des frictions. Le délai observé dans l'ajustement des prix à la suite d'un choc pourrait être expliqué par l'illusion monétaire. Les croyances subjectives selon lesquelles les autres agents ne répondent pas aux chocs peuvent aussi l'expliquer. ${ }^{17}$ Dans des jeux avec complémentarités stratégiques, qui caractérisent un certain nombre de situations macroéconomiques, ces deux explications se renforcent l'une l'autre et peuvent amplifier les effets réels de la politique monétaire. Par ailleurs, l'étude en laboratoire du processus de formation des anticipations nous apprend que les sujets adaptent leurs anticipations lentement, ce qui peut aussi expliquer la persistance des effets réels. Il est utile de prendre en compte ces différentes formes de non-neutralité monétaire et donc ces différents canaux de transmission de la politique monétaire pour étudier la façon dont les banques centrales doivent prendre leurs décisions et en particulier pour déterminer des règles de politique monétaire efficaces. Un certain nombre d'études expérimentales se sont précisément penchées sur ces questions.

\section{Décisions de politique monétaire}

Nous présentons dans cette section les résultats des expériences liées au processus de décision des banques centrales, c'est-à-dire en matière de fixation des règles de politique monétaire mais aussi en ce qui concerne la prise de décisions au sein de groupes que sont les comités de politique monétaire. En effet, une évolution majeure dans la prise de décision au niveau des banques centrales est le passage de la décision individuelle à la décision de groupe. La prise de décision varie considérablement d'un comité de politique monétaire à l'autre. L'expérimentation permet d'apporter un éclairage sur la façon optimale de former des comités de politique monétaire pour obtenir une prise de décision efficace. 
Comment les sujets jouant le rôle de banquiers centraux choisissent-ils les règles monétaires et prennent-ils leurs décisions? S'il n'est pas question ici de considérer que les sujets participants à une expérience se comportent comme de parfaits banquiers centraux, la question est de savoir s'il existe des environnements, des instruments ou des heuristiques qui aident les sujets à prendre des décisions qui sont supposées être importantes dans les modèles de politique monétaire.

\section{Règles monétaires}

En ce qui concerne la conception de règles monétaires tout d'abord, Engle-Warnick et Turdaliev [2010] étudient de manière explicite comment les sujets choisissent des règles de politique monétaire.

Ils considèrent un modèle de type DSGE avec deux variantes. Dans ce qu'ils appellent le modèle 1, l'inflation dépend de la production présente et de l'inflation passée. Dans leur modèle 2, l'inflation courante est également directement affectée par l'inflation prévalent deux périodes auparavant. La tâche des sujets consiste à stabiliser l'économie en fixant un taux d'intérêt. L'expérience est décontextualisée, sa conception est très proche de celle de Duffy et Heinemann : deux containers, A (interprété par Engle-Warnick et Turdaliev comme le produit) et B (interprété comme l'inflation) sont remplis de jetons ; accroître l'instrument revient à réduire le nombre de jetons. Le but des sujets est de maintenir le niveau de jetons dans le container B le plus près possible de 5 . Leurs gains dépendent de leur proximité à cette cible sur les 50 périodes du jeu.

Engle-Warnick et Turdaliev montrent que la plupart des banquiers centraux expérimentaux parviennent à stabiliser l'inflation avec succès en changeant le taux d'intérêt de manière beaucoup plus graduelle que la politique optimale. Ils lissent le taux d'intérêt face à une situation d'incertitude. Les auteurs montrent aussi que les banquiers centraux les plus efficaces utilisent la règle de Taylor.

Noussair, Pfajfar et Zsiros [2011] se concentrent sur les interrelations entre plusieurs marchés : ils présentent un véritable DSGE en laboratoire. ${ }^{18}$ En ce qui nous concerne ici, ils analysent notamment la façon dont les sujets prenant le rôle de banquiers centraux parviennent à stabiliser l'inflation. Ils montrent que la plupart des sujets contrôlent relativement bien l'inflation et que des règles de type règle de Taylor donnent une bonne description des décisions prises par les sujets. Les banquiers centraux expérimentaux génèrent toutefois une plus grande persistance, une production plus faible et un bien-être inférieur à ce qui serait généré dans le même jeu par un automate qui suivrait la règle de Taylor.

\section{Règles de prise de décision au sein de comités de politique monétaire}

La taille et la structure des comités de politique monétaire font l'objet de débats. La prise de décision au sein de comités est quelque chose de commun au sein des banques centrales. La composition et les règles de décision au sein d'un comité peuvent affecter l'issue de ses réunions/délibérations et la qualité des décisions prises. Les règles de décisions des comités de politique monétaire varient considérablement d'une banque centrale à une autre. ${ }^{19}$ 
Il existe de nombreuses expériences dans des champs extérieurs à celui des banques centrales, qui montrent que de petits groupes parviennent à prendre de meilleures décisions que des individus isolés confrontés au même problème. Les groupes sont moins rapides pour prendre des décisions. Tandis que la durée d'une réunion d'un comité de politique monétaire (mesurée en heures) est non pertinente du point de vue des performances macroéconomiques, le nombre de réunions dont un comité a besoin pour se mettre d'accord sur un changement de taux d'intérêt en réponse à un choc perçu est davantage de l'ordre de semaines voire de mois et peut avoir des conséquences macroéconomiques. Il existe d'autres différences importantes entre les décisions de politique monétaire et les tâches auxquelles sont habituellement confrontés les participants à ce type d'expériences. En particulier, lors de la prise de décision en matière de politique monétaire, l'instrument n'affecte les données macroéconomiques qu'avec un important délai. De plus, les décisions sont prises en information incomplète. Les groupes sont-ils plus efficaces pour traiter ce type de questions?

Blinder et Morgan [2005, 2008] ainsi que Lombardelli, Proudman et Talbot [2005] analysent l'efficacité des décisions individuelles vs. en comité au moyen d'expériences de laboratoire. Les premiers sélectionnent des étudiants qui ont suivi au moins un cours de macroéconomie pour jouer le rôle de banquiers centraux prenant des décisions de politique monétaire, soit individuellement soit collectivement. Chaque ordinateur est programmé selon un modèle avec une courbe de Phillips standard telle que l'inflation dépend des déviations du taux de chômage retardé par rapport à son niveau naturel présumé de 5\%, et de ses 4 propres valeurs retardées, dont la somme des poids est égale à 1 ; et une courbe IS dans laquelle le taux de chômage remplace le produit réel : le chômage tend à augmenter au-delà (ou tomber en dessous) de son taux naturel lorsque le taux d'intérêt réel est audessus (respectivement en dessous) de sa valeur «neutre», qui est aussi de 5\%. Mais il y a un retard dans la relation de sorte que le chômage répond au taux d'intérêt réel seulement graduellement. Comme les banquiers centraux du monde réel, les sujets contrôlent seulement le taux d'intérêt nominal. Les paramètres du modèle étaient choisis de telle sorte que l'économie considérée ressemble à l'économie américaine. Les sujets étaient supposés réagir à un choc de demande. Deux chocs stochastiques temporaires rendaient la détection du choc permanent non triviale. La durée considérée était de 20 périodes et les auteurs ont expliqué aux sujets de considérer chaque période comme un trimestre, de sorte qu'au total l'expérience simulait une économie sur une durée de 5 ans. Les gains par période étaient donnés par une fonction de perte linéaire tenant compte des déviations du chômage et de l'inflation par rapport à leurs valeurs ciblées. Pour atteindre leurs cibles, les sujets pouvaient changer le taux d'intérêt à toute période (moyennant un coût fixe de 10 points). Leur tâche principale était de reconnaître un choc permanent dans les données relatives au chômage et à l'inflation et d'y répondre de façon appropriée.

Chaque session expérimentale comprenait 40 périodes. Lors des 10 premières, les sujets jouaient seuls. Lors des 10 suivantes, ils étaient appariés en groupes de 5. Lors des 10 suivantes, les sujets jouaient à 
nouveau seuls. Lors des 10 dernières, ils étaient à nouveau appariés en groupes de 5. L'expérience comportait 20 sessions. Dans dix d'entre elles, les décisions de groupe se faisaient à la majorité ; dans les 10 autres, elles se prenaient à l'unanimité.

Le principal résultat de Blinder et Morgan [2005] est le suivant: les groupes ont pris de meilleures décisions que les individus, sans pour autant avoir eu besoin de davantage de temps. Le temps mis pour prendre une décision était mesuré par le nombre de périodes (c'est-à-dire la quantité de données nécessaires pour qu'un individu ou un groupe décide de changer le taux d'intérêt après l'occurrence d'un choc). Selon Blinder et Morgan [2005, p. 801] : “dans la plupart des cas, une fois que trois ou quatre sujets s'étaient mis d'accord sur la marche à suivre, l'autre ou les deux autres sujets les suivaient immédiatement" ${ }^{20}$.

Dans la réalité, la taille des comités varie largement d'un comité de politique monétaire à l'autre. Par ailleurs, il existe généralement un leader au sein des comités de politique monétaire (par exemple le gouverneur dans le cas de la Fed). Blinder et Morgan [2008] analysent expérimentalement la pertinence de la taille du comité (plus précisément, ils comparent la performance relative de petits et de grands groupes) ainsi que l'importance d'avoir un leader au sein du groupe (plus précisément, ils veulent savoir si les groupes prennent de meilleures décisions en présence d'un leader).

Chaque groupe est composé de 4 ou 8 sujets. Un leader est désigné dans la moitié des sessions. Son vote est décisif et son score est doublé. Les auteurs montrent que des groupes plus grands ont de meilleures performances, mais que le fait que le groupe ait un leader ou non n'a pas d'impact significatif sur la performance. La taille du groupe n'impacte pas ce résultat.

Maier [2010] s'intéresse au système de rotation mis en place à la fois par la Banque Centrale Européenne et la Réserve Fédérale Américaine pour limiter le nombre de membres votants (i.e. le droit de vote tourne selon une séquence prédéterminée). Les membres non-votants ne prennent quasiment jamais part à la discussion et donc la rotation aide à accroître la quantité d'information sans changer la taille du groupe. Cependant, si les intérêts des membres du comité ne sont pas pleinement alignés, les membres votants peuvent exploiter le fait que certains membres ne votent pas. Selon Bosman et al. [2013], les membres d'un comité peuvent être confrontés à un dilemme du prisonnier : chaque membre voterait de sorte à maximiser ses gains. Un tel comportement individualiste pourrait conduire les comités à prendre des décisions moins bonnes que si chaque membre avait simplement voté pour l'option qui maximise les gains du groupe dans son ensemble.

Cette partie s'est focalisée sur les études expérimentales relatives aux décisions de politique monétaire. Ces expériences ont ainsi porté d'une part sur les règles de politique monétaire et d'autre part sur la prise de décision au sein des comités de politique monétaire. Elles montrent que l'utilisation de règles est assez naturelle. En particulier, les comportements observés tendent vers la règle de Taylor. Les expériences permettent aussi d'établir la structure optimale des comités de politique 
monétaire et mettent notamment en évidence la supériorité de la prise de décision (en matière de politique monétaire) en groupe. Ainsi, même si l'on ne s'attend bien évidemment pas à ce que les participants aux expériences (qui sont la plupart du temps des étudiants) parviennent à atteindre les objectifs de politique monétaire comme le feraient des banquiers centraux professionnels, il semble que les comportements observés tant dans l'utilisation de règles que dans la prise de décision en groupe se rapprochent assez naturellement des pratiques des banquiers centraux. En testant différents aspects de la prise de décision de manière isolée, on peut identifier certains aspects de ce processus de prise de décision qui sont particulièrement demandant et analyser dans le laboratoire quels types de biais, d'heuristiques et d'erreurs rendent cette prise de décision délicate. Ces résultats peuvent être robustes et tenir dans des situations où la prise de décision est plus complexe, en présence de professionnels rôdés à ce type de décision, dans la mesure où ils décrivent des aspects généraux de la prise de décision. Par ailleurs, une partie de la littérature relative à la politique monétaire suppose que les banquiers centraux répondent à des incitations. Dans le laboratoire, les sujets répondent précisément à ces incitations. De ce fait, le laboratoire représente un environnement dans lequel il est possible de tester si un système d'incitation a bien les effets attendus sur les comportements. Une fois la prise de décision de politique monétaire étudiée en laboratoire, se pose la question de sa mise en œuvre.

\section{Mise en ouvre de la politique monétaire}

Nous analysons maintenant la façon dont l'économie expérimentale étudie la mise en œuvre de la politique des banques centrales. A cet égard, il est important de considérer la façon dont les banques centrales communiquent leurs décisions. La communication est un outil important à la disposition des banques centrales. Par ailleurs, un autre aspect de la mise en œuvre de la politique monétaire concerne la façon dont les banques centrales pratiquent le refinancement des banques par appel d'offre. Nous analysons successivement ces deux questions.

\section{Transparence et communication des banques centrales}

Depuis une vingtaine d'années, on assiste à une plus grande transparence de la part des banques centrales. Cet accroissement est notamment allé de paire avec l'adoption par de nombreuses banques centrales de régimes de ciblage d'inflation (Nouvelle-Zélande, Canada, Royaume-Uni et Suède au début des années 1990). La transparence réduit, voire élimine les asymétries d'information entre la banque centrale et le secteur privé/les participants au marché. Pourtant un certain nombre d'auteurs montrent les limites potentielles d'une transparence accrue. En effet, il existe un débat quant aux effets stabilisants ou non de la transparence. Les marchés financiers sont connus pour réagir fortement aux annonces publiques et tout particulièrement aux conférences de presse ou aux discours diffusés par les banques centrales. Selon Morris and Shin [2002], les complémentarités stratégiques entre les actions des participants au marché, inhérentes aux modèles macroéconomiques, donnent des incitations aux 
agents à sur-réagir aux annonces publiques par rapport au contenu informationnel de ces annonces sur les fondamentaux économiques. La sur-réaction peut être définie dans leur modèle comme le fait que le poids attribué au signal public dans l'action d'équilibre est supérieur à sa précision relative sur les fondamentaux. Cette sur-réaction est liée au fait que les signaux publics contiennent davantage d'information (que les signaux privés) sur les croyances des autres agents. Ils montrent ainsi que les annonces publiques peuvent être dommageables en termes de bien-être en distordant l'action d'équilibre par rapport à celle qui serait justifiée par les fondamentaux.

Cornand et Heinemann [2014a] proposent une mesure expérimentale de la sur-réaction des agents aux signaux publics dans un jeu de coordination proche du cadre de Morris et Shin. Ils montrent que lorsque l'utilité des sujets dépend à la fois d'un motif fondamental et d'un motif de coordination, ces derniers attribuent un poids plus fort au signal public que celui qui serait justifié par son seul contenu informationnel sur le fondamental, mais ce poids est inférieur à celui théoriquement prédit. Dans un cadre proche, Dale et Morgan [2012] analysent l'impact de la précision des signaux sur les gains des sujets et mettent en évidence les effets en bien-être issus de la sur-réaction aux signaux public. Ils montrent que l'ajout d'un signal privé de pauvre qualité améliore la qualité des décisions. Par contre, lorsqu'il s'agit de l'ajout d'un signal public de pauvre qualité, les sujets placent un poids trop important sur le signal public ce qui détériore le bien-être.

Tandis que les expériences précédentes ne formulaient pas véritablement de marché financier, Ackert, Church et Gillette [2004] présentent une expérience de laboratoire dans laquelle ils considèrent un marché d'actifs ; des traders reçoivent des signaux publics de qualités différentes (mais pas de signaux privés). Ils montrent que les traders sur-réagissent à l'information publique de mauvaise qualité et sous-réagissent à l'information publique de bonne qualité.

Alfarano et al. [2011] analysent dans quelle mesure le processus d'agrégation de l'information privée sur un marché peut pâtir de la présence de signaux publics (fournis par exemple par des agences de notation). Chaque agent est doté d'un certain nombre d'unités d'actifs donnant un dividende en fin de période et d'un autre montant de monnaie expérimentale (à chaque période, le dividende est déterminé de manière aléatoire par l'expérimentaliste et n'est pas connu des agents avant la fin de la période). A chaque période, les sujets forment des offres et des demandes d'actifs, ou acceptent directement des offres et demandes des autres traders. Pour prendre leurs décisions, les sujets pouvaient acheter autant de signaux privés qu'ils le souhaitaient à propos du dividende pendant toute la durée de la période (et tant qu'ils avaient suffisamment de monnaie expérimentale). Dans le traitement avec information publique, les sujets avaient aussi accès sans coût à un signal public sur le dividende de l'actif. Les auteurs montrent qu'il existe un effet d'éviction : lorsque l'information est diffusée, les sujets achètent moins de signaux privés.

Middeldorp et Rosenkranz [2011] considèrent également un marché d'actifs expérimental avec information privée coûteuse, proche de Diamond [1985]. Leurs résultats confirment la théorie ainsi 
que les expériences précédentes : un signal public plus précis de la part de la banque centrale peut dans certains cas évincer l'acquisition d'information privée. Le marché n'est pas toujours en mesure d'agréger l'information privée, ce qui peut conduire à une détérioration de la qualité informationnelle du marché, qui à terme peut réduire la capacité du marché à prédire la politique monétaire future.

Ces expériences mettent ainsi en évidence expérimentalement la sur-réaction aux annonces publiques et le fait que cette sur-réaction est dommageable.

Dans la mesure où la sur-réaction aux annonces publiques est dommageable en termes de bien-être, il est important de se demander si les banques centrales peuvent réduire la sur-réaction à leurs annonces. Baeriswyl et Cornand [2014] testent précisément en laboratoire les stratégies de communication à même de réduire la sur-réaction du marché aux annonces publiques. Ils considèrent deux stratégies de communication. La première est la publicité partielle, c'est-à-dire le fait de diffuser un signal semipublic, commun à une fraction des participants au marché seulement. Choisir un canal de communication qui n'atteint pas tous les participants au marché permet de réduire la sur-réaction car les agents non informés ne réagissent tout simplement pas, tandis que les agents informés réagissent moins fortement puisqu'ils savent que certains agents ne sont pas informés. La seconde stratégie de communication correspond à la transparence partielle, c'est-à-dire le fait de diffuser de l'information publique avec une certaine ambigüité, mais à l'ensemble des participants au marché cette fois-ci. Communiquer avec une certaine ambigüité réduit la sur-réaction dans la mesure où l'ambigüité conduit les participants au marché à ne pas être certains de la façon dont les autres agents interprètent un même signal, limitant ainsi le potentiel focal de l'annonce. Les auteurs montrent qu'en théorie les deux stratégies de communication sont équivalentes pour limiter la sur-réaction aux signaux publics, dans le sens où un signal avec degré limité de publicité ou un signal avec degré de transparence limité peuvent conduire à la même action moyenne de la part des participants, et donc au même niveau de bien-être. Tandis que la publicité partielle réduit la sur-réaction seulement dans la mesure où les sujets non informés ne réagissent pas au signal public (les sujets informés eux se conduisant comme si l'information était totalement publique au sein de l'ensemble des participants), la transparence partielle réduit la sur-réaction car l'ambigüité conduit les sujets à se comporter de manière prudente. En pratique, les auteurs concluent que les banques centrales devraient privilégier la transparence partielle dans la mesure où elle ne pose pas de problème d'équité et n'est pas sujette aux problèmes liés à la diffusion d'information qui touchent la publicité partielle. ${ }^{21}$ Une banque centrale qui cherche à limiter la sur-réaction à ses annonces devrait dès lors formuler le contenu de ses annonces de manière à éviter un fort potentiel focal plutôt que de sélectionner son audience.

Cornand et M'baye [2013] font le lien entre communication et politique de stabilisation en laboratoire. Ils présentent une expérience fondée sur un modèle nouveau keynésien pour tester la pertinence de différents régimes de ciblage d'inflation. Ils évaluent dans quelle mesure la communication de la cible d'inflation est importante pour le succès de ce régime à stabiliser l'inflation, mais aussi en termes de 
stabilisation de la production (de ce point de vue, ils évaluent aussi dans quelle mesure les objectifs de la banque centrale affectent ces performances). Un régime de ciblage d'inflation est caractérisé par l'annonce explicite d'une cible d'inflation numérique ou d'une bande, un mandat clair pour la banque centrale dont l'objectif premier est la stabilisation de l'inflation, un fort degré de transparence (voir e.g. Svensson [2010]). Empiriquement, on constate une forte hétérogénéité des régimes de ciblage d'inflation $(\mathrm{CI})$, selon le degré d'application de ces trois critères. Les bénéfices liés à l'adoption d'un régime de ciblage d'inflation explicite font encore l'objet de débats. ${ }^{22}$

Le modèle est fondé sur 3 équations de base : une équation de demande agrégée (courbe IS), une fonction d'offre (courbe de Phillips de type Calvo [1983]), une fonction de réaction de la banque centrale (règle de taux d'intérêt). L'expérience consiste à collecter les anticipations d'inflation des sujets en laboratoire et à les réinsérer dans le modèle théorique pour dériver les valeurs courantes de l'inflation, de la production et du taux d'intérêt. ${ }^{23}$ Période après période, les séries temporelles des principales variables sont générées.

Les auteurs ont considéré quatre traitements. Dans le traitement CI implicite strict, le seul objectif de la banque centrale est de stabiliser l'inflation, mais la cible n'était pas annoncée. Dans le traitement CI explicite strict, la banque centrale avait aussi pour seul objectif de stabiliser l'inflation (à 5\%), mais communiquait explicitement sa cible par une annonce. Dans le traitement CI implicite flexible, la banque centrale avait deux objectifs, l'un d'inflation et l'autre de production et n'annonçait pas sa cible au public. Dans le traitement CI explicite flexible, la banque centrale avait également deux objectifs, mais communiquait explicitement la valeur de sa cible d'inflation. La tâche des sujets consistait à prévoir l'inflation de la période suivante pour chacune des 60 périodes d'une session. $\mathrm{Au}$ total, l'expérience comptait 4 sessions (comprenant 6 sujets) par traitement. Les instructions présentaient l'information générale sur les variables composant l'économie (inflation, production, taux d'intérêt, et cible d'inflation selon les traitements). Les participants savaient que les valeurs courantes de l'inflation et de la production dépendaient principalement de leurs propres prévisions et des prévisions des autres. Les gains étaient tels que les participants obtenaient des points lorsque leur erreur de prévision était inférieure à $3 \%$.

Les auteurs montrent que si la banque centrale cherche seulement à stabiliser l'inflation, annoncer ou non la cible n'a pas d'impact en termes de performances macroéconomiques : une politique monétaire active est suffisante pour obtenir les mêmes performances macroéconomiques (voir Figure 4). Au contraire, si la banque centrale cherche aussi à stabiliser l'économie, communiquer sa cible permet de réduire la volatilité de l'inflation, du taux d'intérêt et de la production sans affecter le niveau de ces variables. La pertinence de l'annonce de la cible est due à la baisse de l'incertitude sur ses objectifs. 


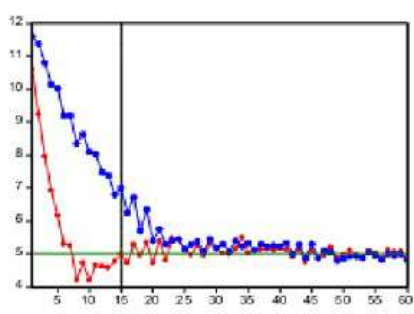

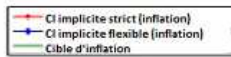

Ciblage d'inflation explicite strict versus explicite flexible
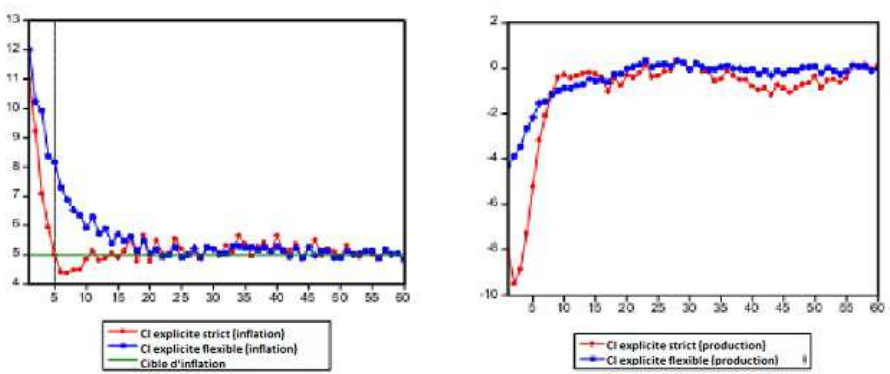

Figure 4 : Séries moyennes d'inflation et de production pour des régimes de ciblage d'inflation strictes versus flexibles - Source : Cornand et M'baye [2013].

Ainsi, la question de la communication doit être établie conjointement à la politique de stabilisation de la banque centrale.

La question de la communication en période de crise a aussi été traitée du point de vue expérimental. Lorsqu'une économie fait face à une crise de change ou une crise bancaire, la communication a des effets qui sont distincts de ceux qu'elle a en temps normal car les pures crises de liquidité sont des phénomènes auto-réalisateurs avec équilibres multiples (au sens où leur occurrence peut ou non se produire ex ante).

Morris et Shin [1998, 2003] et Heinemann et Illing [2002] appliquent la théorie des jeux globaux à un modèle de crise de change ${ }^{24}$ et montrent qu'accroître la transparence sur les fondamentaux économiques augmente la probabilité d'attaque. ${ }^{25}$ Heinemann, Nagel et Ockenfels [2004] testent ce modèle d'attaque spéculative et montrent que l'information commune ne conduit pas à une plus faible prévisibilité dans les comportements associée à l'existence d'équilibres multiples. Même si l'information est publique, les sujets se comportent comme si ils recevaient des signaux privés. ${ }^{26}$ Toutefois, ils montrent que les attaques spéculatives sont plus probables lorsque les fondamentaux sont transparents. ${ }^{27}$ Ainsi, en période de crise, l'information est susceptible d'être fortement déstabilisante.

\section{Refinancement et enchères}

Du point de vue opérationnel, des opérations de refinancement (open-market) sont menées pour assurer la liquidité du système financier. En particulier la Banque Centrale Européenne conduit des 
appels d'offre (repo auctions) sur une base hebdomadaire. La Réserve Fédérale Américaine fait des appels d'offre quotidiens. Par le biais de ces appels d'offre, une banque centrale offre de la liquidité aux banques qui en ont besoin pour refinancer leurs obligations. Comment ces appels d'offre sont-ils formulés ? Sont-ils efficaces ? Les enchères sont un sujet classique en économie expérimentale. Mais les tests expérimentaux concernant directement les appels d'offre de refinancement sont rares.

Ehrhart [2001] étudie le mécanisme d'appel d'offre à taux fixe (fixed rate tender mechanism ou adjudication «à la hollandaise ») utilisé par la Banque Centrale Européenne (BCE) avant 2000. Le mécanisme est le suivant. La BCE fixe par avance un taux d'intérêt auquel elle est prête à faire des transactions. Les banques soumettent des offres pour des montants de liquidité qu'elles souhaitent emprunter à ce taux. Si la somme des offres est trop élevée, les banques sont rationnées de manière proportionnelle à leurs offres. Dans la mesure où ce système crée une incitation des banques à exagérer leurs besoins de refinancement, on a observé une hausse continue des offres. Une telle incitation est problématique car il devient difficile pour la banque centrale d'extraire de l'information sur les besoins de liquidité des banques alors même que les opérations de refinancement sont supposées aider la banque centrale à évaluer et prévoir la politique monétaire. C'est la raison pour laquelle la BCE est passée à un mécanisme de type interest-rate tender (ou adjudication «à l'américaine ») qui ne souffre pas d'explosions du nombre d'offres, mais présente l'inconvénient d'inciter à la sous-enchère.

Ehrhart cherche à évaluer si et dans quelles conditions un appel d'offre à taux fixe conduit à une augmentation stratégique des offres et comment cela affecte le contenu informationnel de ces offres. Son expérience consiste à répéter plusieurs jeux d'appels d'offre à taux fixe. Les sujets représentent des banques (la banque centrale étant jouée par l'ordinateur). Il montre qu'une tendance explosive dans les offres ne peut pas être stoppée en changeant simplement les règles de distribution, ni les valeurs d'équilibre. Une fois que les offres ont perdu leur contenu informationnel, elles demeurent non informatives car les offreurs répondent plus fortement à des considérations stratégiques qu'à leurs propres besoins.

En matière de mise en œuvre de la politique monétaire, deux aspects ont été considérés : les questions de communication des banques centrales et, plus modestement, les mécanismes d'appel d'offre en matière de refinancement. Dans ces deux domaines, les expériences de laboratoire sont tout particulièrement utiles. En effet, l'impact de l'information et les canaux de communication se prêtent bien à expérimentation car l'expérimentateur est à même de contrôler l'information que reçoivent les participants aux expériences et peut aisément distinguer différents canaux de communication dans différents traitements. Tandis que l'analyse des questions informationnelles sur données réelles pâtit de l'existence simultanée de différents canaux de communication et de problèmes liés au filtrage de l'information qui a effectivement affecté les décisions, les expériences permettent d'obtenir des 
résultats clairs. Les expériences relatives à la communication des banques centrales ont ainsi mis en évidence la sur-réaction des agents aux annonces et montrent que certaines stratégies de communication peuvent en limiter leurs effets, en temps normal et en temps de crise. Elles montrent également qu'il est nécessaire de considérer conjointement la politique de communication et la politique de stabilisation. La conception des mécanismes d'enchères se prête elle-aussi très bien à l'expérimentation et est d'ailleurs dorénavant un thème classique en économie expérimentale. Son utilisation pourrait être davantage développée aux questions de refinancement.

\section{Conclusion et perspectives}

Cet article a passé en revue la littérature relative aux applications de l'expérimentation à l'étude des fonctions des banques centrales. Il a montré que les expériences de laboratoire peuvent être utiles pour mieux comprendre les canaux par lesquels la politique monétaire affecte les décisions, l'impact des stratégies de communication qui sont aujourd'hui au cœur des préoccupations des banques centrales ainsi que la façon dont elle peuvent contribuer à tester l'efficacité de certaines politiques. Plus précisément, il est possible de mettre tout d'abord en évidence expérimentalement différents canaux par lesquels la politique monétaire affecte les comportements et notamment celui des anticipations. Les délais d'ajustement de prix observés à la suite de chocs peuvent être expliqués en partie par l'illusion monétaire, les croyances subjectives selon lesquelles les autres agents ne répondent pas aux chocs pouvant représenter une explication complémentaire. En particulier, lorsqu'une situation peut être modélisée par un jeu avec complémentarités stratégiques, comme cela est souvent le cas en macroéconomie, ces explications se renforcent l'une et l'autre. Il est également possible de rendre compte des délais d'ajustement dans les prix grâce aux modèles fondés sur les prix rigides ou l'information rigide. L'étude de la formation des anticipations en laboratoire montre aussi d'une part que le biais inflationniste peut être mis en évidence expérimentalement et que seul l'engagement permet de le limiter. D'autre part, cette littérature souligne que les sujets ont généralement un comportement adaptatif qui peut expliquer les phénomènes de persistance. Il est nécessaire de tenir compte de ces différentes formes de non-neutralité monétaire pour en déduire des règles de politique monétaire efficaces. Deuxièmement, les expériences en matière de prise de décisions de politique monétaire (règles de politique monétaire et prise de décision au sein des groupes que sont les comités de politique monétaire) permettent de montrer que l'utilisation de règles est assez naturelle, en particulier, les comportements tendent vers la règle dite de Taylor. Les expériences permettent aussi d'établir la structure optimale des comités de politique monétaire. Troisièmement, en matière de mise en œuvre de la politique monétaire, les expériences relatives à la communication des banques centrales mettent en évidence la sur-réaction des agents aux annonces et montrent que certaines stratégies de communication peuvent en limiter leurs effets, en temps normal et en temps de crise. 
Il est nécessaire de souligner que les expériences de laboratoire doivent être considérées comme complémentaires des autres approches empiriques utilisées en macroéconomie. Le fait que les expériences puissent être répliquées (de façon à créer un grand nombre d'économies aux caractéristiques similaires et donc à collecter un grand nombre d'observations) en fait un outil approprié pour traiter des questions de politique économique. Bien que les économies générées soient synthétiques, elles peuvent conserver les caractéristiques principales des économies réelles, permettant ainsi de répondre à des questions de recherche spécifiques. Les réponses que peut apporter l'économie expérimentale du point de vue du champ de la macroéconomie sont pertinentes car le laboratoire fournit un environnement contrôlé permettant de tester de manière alternative et répétée différentes mesures de politique économique et de régulation financière.

De nombreux autres domaines autour des fonctions des banques centrales pourraient bénéficier de l'expérimentation. En particulier, la crise récente a initié un débat sur l'étendue des fonctions des banques centrales [Eichengreen et al., 2011]. L'expérimentation peut représenter un outil pour redéfinir ces fonctions. Premièrement, comment la dette et les pressions politiques affectent-elles les objectifs des banques centrales? Deuxièmement, comment l'ajout d'objectifs supplémentaires comme les objectifs de change affectent-ils les fonctions des banques centrales? L'ajout d'objectifs supplémentaires peut en particulier accroître les complémentarités stratégiques entre les actions des participants au marché et rendre l'économie plus sujette aux attaques spéculatives. Troisièmement, quelle est la relation entre stabilité des prix et stabilité financière ? La crise a montré que la séparation traditionnelle entre autorités monétaires visant la stabilité des prix et autorités de régulation visant la stabilité financière de manière indépendante n'était plus viable. L'expérimentation pourrait permettre d'analyser comment les règles de politique monétaire et la régulation financière interagissent pour contenir les bulles sur les prix d'actifs. Quatrièmement, elle pourrait aussi permettre de tester si la gestion des prix d'actifs grâce à la politique monétaire est compatible avec un objectif d'inflation. Si tel n'est pas le cas, il serait aussi possible de tester l'impact de ratios prudentiels sur la stabilité financière et plus généralement de la supervision macro-prudentielle et de la supervision du système bancaire. Cinquièmement, elle pourrait permettre de tester les mesures de politique nonconventionnelle. La crise a montré que les instruments traditionnels de politique monétaire pouvaient devenir inefficaces. Parce que les mesures non-conventionnelles ont été adoptées dans des circonstances bien particulières, les données réelles n'offrent que des illustrations très spécifiques de leurs effets. Au contraire, les expériences de laboratoire permettent de les analyser plus systématiquement et d'isoler leurs effets.

\section{Références bibliographiques}

L. Ackert, B. Church et A. Gillette [2004] : Immediate disclosure or secrecy? The release of information in experimental asset markets, Financial Markets, Institutions and Instruments, vol. 13, n $^{\circ}$, p. 219-243. 
K. Adam [2005] : Learning to forecast and cyclical behavior of output and inflation, Macroeconomic Dynamics, vol. $9, \mathrm{n}^{\circ} 1$, p. 1-27.

K. Adam [2007a]: Experimental Evidence on the Persistence of Output and Inflation, Economic Journal, vol. 117, p. 603-635.

K. Adam [2007b] : Optimal Monetary Policy with Imperfect Common Knowledge, Journal of Monetary Economics, vol. 54, n², p. 276-301.

S. Alfarano, A. Morone et E. Camacho [2011] : The role of public and private information in a laboratory financial market, Working Papers Serie AD 2011-06, Instituto Valenciano de Investigaciones Económicas, S.A. (Ivie).

R. Amano, J. Engle-Warnick et M. Shukayev [2011] : Price-level targeting and inflation expectations: experimental evidence, Bank of Canada Working Paper 2011-18.

A. Angeriz et_P. Arestis [2008] : Assessing Inflation Targeting Through Intervention Analysis, Oxford Economic Papers, vol. 60, n², p. 293-317.

M. Anufriev, T. Assenza, C. Hommes et D. Massaro [2013] : Interest Rate Rules with Heterogeneous Expectations, Macroeconomic Dynamics, vol. 31, p. 1574-1604.

J. Arifovic et J. H. Jiang [2013]: Experimental Evidence of Sunspot Bank Runs, mimeo Bank of Canada.

J. Arifovic et T. J. Sargent [2003] : Laboratory Experiments with an Expectational Phillips Curve, in D. E. Altig et B. D. Smith [eds.], Evolution and Procedures in Central Banking, Cambridge University Press, Cambridge.

T. Assenza, T. Bao, C. Hommes et D. Massaro [2014] : Experiments on Expectations in Macroeconomics and Finance, in J. Duffy (ed.) Experiments in Macroeconomics (Research in Experimental Economics, Volume 17) Emerald Group Publishing Limited, p. 11-70.

R. Baeriswyl et C. Cornand [2014] : Reducing overreaction to central banks disclosure: theory and experiment, Journal of the European Economic Association, vol. 12, n 4, p. 1087-1126.

T. Bao, C. Hommes, J. Sonnemans et J. Tuinstra [2012] : Individual Expectations, Limited Rationality and Aggregate Outcomes, Journal of Economic Dynamics and Control, vol. 36, p. 1101-1120.

L. Ball et N. Sheridan [2005] : Does Inflation Targeting Matter?, in B. Bernanke et M. Woodford [eds.], The Inflation Targeting Debate, University of Chicago Press, p. 249-276.

R. Barro et D. Gordon [1983] : A Positive Theory of Monetary Policy in a Natural Rate Model, Journal of Political Economy, vol. 91, n4, p. 589-610.

M. Bernasconi et O. Kirchkamp [2000] : Why do monetary policies matter? An experimental study of saving and inflation in an overlapping generations model, Journal of Monetary Economics, vol. 46, p. 315-343.

A. S. Blinder et J. Morgan [2005] : Are Two Heads Better than One? Monetary Policy by Committee, Journal of Money, Credit, and Banking, vol. 37, n5, p. 789-812.

A. S. Blinder et J. Morgan [2008] : Leadership in Groups: A Monetary Policy Experiment, International Journal of Central Banking, vol. 4, nº 4, p. 117-150.

A. S. Blinder et C. Wyplosz [2005] : Central Bank Talk: Committee Structure and Communication Policy, ASSA meetings, Philadelphie, janvier.

R. Bosman, P. Maier, V. Sadiraj et F. van Winden [2013] : Let Me Vote! An experimental study of the effects of vote rotation in committees, Journal of Economic Behavior and Organization, vol. 96, $\mathrm{n}^{\circ} \mathrm{C}, \mathrm{p} .32-47$.

G. Calvo [1983] : Staggered prices in a utility maximizing framework, Journal of Monetary Economics, vol. 12, p. 383-398. 
A. Cheremukhin, Popova A. et Tutino A. [2011] : Experimental Evidence on Rational Inattention, Federal Reserve Bank of Dallas Working Paper 1112.

L. J. Christiano, M. Eichenbaum et C. Evans [2005] : Nominal rigidities and the dynamic effects of a shock to monetary policy, Journal of Political Economy, vol. 113, p.1-45.

R. Cooper et A. John [1988] : Coordinating Coordination Failures in Keynesian Models, Quarterly Journal of Economics, vol. 103, n³, p. 441-463.

C. Cornand et F. Heinemann [2014a] : Measuring agents' overreaction to public information in games with strategic complementarities, Experimental Economics, vol. 17, p. 61-77.

C. Cornand et F. Heinemann [2014b] : Experiments on monetary policy and central banking, in Experiments in Macroeconomics, J. Duffy [eds.], vol. 17, Research in Experimental Economics, Emerald Press.

C. Cornand et C.K. M'baye [2013] : Does inflation targeting matter? An experimental investigation, Working paper GATE 2013-30.

D. J. Dale et J. Morgan [2012] : Experiments on the Social Value of Public Information, mimeo.

D. Davis et O. Korenok [2011] : Nominal price shocks in monopolistically competitive markets: An experimental analysis, Journal of Monetary Economics, vol. 58, p. 578-589.

D. W. Diamond [1985] : Optimal release of information by firms, Journal of Finance, vol. 40, nº 4 , p. 1071-1094.

D. W. Diamond et P. H. Dybvig [1983] : Bank runs, deposit insurance, and liquidity, Journal of Political Economy, vol. 91, n³, p. 401-419.

J. Duffy et E. Fisher [2005] : Sunspots in the Laboratory, American Economic Review, vol. 95, p. 510-529.

J. Duffy et F. Heinemann [2013] : Central Bank Reputation, Transparency and Cheap Talk as Substitutes for Commitment: Experimental Evidence, mimeo.

J. Duffy [1998] : Monetary Theory in the Laboratory, Federal Reserve Bank of St. Louis Review, septembre-octobre.

J. Duffy [2008a] : Macroeconomics: A Survey of Laboratory Research, University of Pittsburgh Working Paper 334.

J. Duffy [2008b] : Experimental Macroeconomics, in S.N. Durlauf et L.E. Blume [eds.], The New Palgrave Dictionary of Economics, 2nd Ed., New York: Palgrave Macmillan.

K. M. Ehrhart [2001] : European Central Bank Operations: Experimental Investigation of the Fixed Rate Tender, Journal of International Money and Finance, vol. 20, p. 871-893.

B. Eichengreen, M. El-Erian, A. Fraga, T. Ito, J. Pisani-Ferry, E. Prasad, R. Rajan, M. Ramos, C. Reinhart, H. Rey, D. Rodrik, K. Rogoff, H. S. Shin, A. Velasco, B. Weder di Mauro et Y. Yu [2011] : Rethinking Central Banking, Report of the Committee on International Economic and Policy Reform, September.

J. Engle-Warnick et N. Turdaliev [2010] : An experimental test of Taylor-Type rules with inexperienced central bankers, Experimental Economics, vol. 13, n², p. 146-166.

G. W. Evans et S. Honkapohja [1993] : Adaptive forecasts, hysteresis, and endogenous fluctuations, Economic Review of the Federal Reserve Bank of San Francisco, vol. 1, p. 3-13.

G. W. Evans et S. Honkapohja [2001] : Learning and Expectations in Macroeconomics, Princeton: Princeton University Press.

D. Fehr, F. Heinemann et A. Llorente-Saguer [2013] : The Power of Sunspots, working paper. 
E. Fehr et J.-R. Tyran [2001] : Does Money Illusion Matter?, American Economic Review, vol. 91, p. 1239-1262.

E. Fehr et J.-R. Tyran [2005] : Individual Irrationality and Aggregate Outcomes, Journal of Economic Perspectives, vol. 19, p. 43-66.

E. Fehr et J.-R. Tyran [2008] : Limited Rationality and Strategic Interaction: The Impact of the Strategic Environment on Nominal Inertia, Econometrica, vol. 76, n²2, p. 353-394.

E. Fehr et J.-R. Tyran [2014] : Does Money Illusion Matter?: Reply, American Economic Review, vol. 104, p. 1063-1071.

P. M. Geraats [2002] : Central bank transparency, Economic Journal, vol. 112, p. 532-565.

G. Hammond [2012] : State of the Art of Inflation Targeting - 2012, Handbook n ${ }^{\circ} 29$, Centre for Central banking Studies, Bank of England.

F. Heinemann et G. Illing [2002] : Speculative attacks: Unique sunspot equilibrium and transparency, Journal of International Economics, vol. 58, p. 429-450.

F. Heinemann, R. Nagel et P. Ockenfels [2004] : The Theory of Global Games on Test: Experimental Analysis of Coordination Games with Public and Private Information, Econometrica, vol. 72, p. 1583-1599.

F. Heinemann, R. Nagel et P. Ockenfels [2009] : Measuring Strategic Uncertainty in Coordination Games, Review of Economic Studies, vol. 76, p. 181-221.

C. H. Hommes [2011] : The Heterogeneous Expectations Hypothesis: Some Evidence from the Lab, Journal of Economic Dynamics and Control, vol. 35, nำ p. 1-24.

F. E. Kydland et E. C. Prescott [1979] : Rules Rather than Discretion: The Inconsistency of Optimal Plans, Journal of Political Economy, vol. 85, p. 473-492.

A.T. Levin, F.M. Natalucci et J.M. Piger [2004] : The Macroeconomic Effects of Inflation Targeting, Federal Reserve Bank of St. Louis Review, vol. 86, n4, p. 51-80.

S. Lin et H. Ye [2007] : Does Inflation Targeting Really Make a Difference? Evaluating the Treatment Effect of Inflation Targeting in Seven Industrial Countries, Journal of Monetary Economics, vol. 54, p. 2521-2533.

C. Lombardelli, J. Proudman et J. Talbot [2005] : Committees versus Individuals: An Experimental Analysis of Monetary Policy Decision Making, International Journal of Central Banking, vol. 1, $\mathrm{n}^{\circ} 1$, p. 181-205.

B. Maćkowiak et M. Wiederholt [2009] : Optimal Sticky Prices under Rational inattention, American Economic Review, vol. 99, n³, p. 769-803.

P. Maier [2010] : How central banks take decisions: an analysis of monetary policy meetings, in $\mathrm{P}$. Siklos, M. Bohl et M. Wohar [eds.], Challenges in central banking: The current institutional environment and forces affecting monetary policy, Cambridge University Press.

G. Mankiw et R. Reis [2002] : Sticky information versus sticky prices: a proposal to replace the new Keynesian Phillips curve, Quarterly Journal of Economics, vol. 117, p. 1295-1328.

R. Marimon et S. Sunder [1993] : Indeterminacy of Equilibria in a Hyperinflationary World: Experimental Evidence, Econometrica, vol. 61, n5, p. 1073-1107.

R. Marimon et S. Sunder [1995] : Does a Constant Money Growth Rule Help Stabilize Inflation: Experimental Evidence, Carnegie - Rochester Conference Series on Public Policy, vol. 45, p. 111 156.

M. Middeldorp et S. Rosenkranz [2011] : Central Bank Transparency and the Crowding Out of Private Information in an Experimental Asset Market, Federal Reserve Bank of New York Staff Reports, no. 487, March 2011. 
S. Morris et H. S. Shin [1998] : Unique Equilibrium in a Model of Self-Fulfilling Currency Attacks, American Economic Review, vol. 88, p. 587-597.

S. Morris et H. S. Shin [2002] : Social Value of Public Information, American Economic Review, vol. $92, \mathrm{n}^{\circ} 5$, p. $1522-1534$.

S. Morris et H. S. Shin [2003] : Global Games: Theory and Applications, in M. Dewatripont, L.P. Hansen et S. J. Turnovsky [eds.], Advances in Economics and Econometrics: Theory and Applications, vol. 1, Cambridge University Press, London, p. 56-114.

S. Morris et H. S. Shin [2014] : Risk-Taking Channel of Monetary Policy: A Global Game Approach, Working paper, January.

R. Nagel [1995] : Unraveling in Guessing Games: An Experimental Study, American Economic Review, vol. 85, n5, p. 1313-1326.

C. N. Noussair, D. Pfajfar et J. Zsiros [2011] : Frictions, Persistence, and Central Bank Policy in an Experimental Dynamic Stochastic General Equilibrium Economy, European Banking Center Discussion Paper No. 20 11-006.

L. Petersen et A. Winn [2014] : Does Money Illusion matter?: Comment, American Economic Review, vol. 104, p. 1047-1062.

D. Pfajfar et B. Žakelj [2014] : Experimental Evidence on Inflation Expectation Formation, Journal of Economic Dynamics and Control, vol. 44, p. 147-168

R. Ricciuti [2008] : Bringing macroeconomics into the lab, Journal of Macroeconomics, vol. 30, $\mathrm{n}^{\circ} 1$, p. 216-237.

A. Riedl [2010] : Behavioral and Experimental Economics Do Inform Public Policy, Finanzarchiv, vol. 66, p. 65-95.

S. Roger [2009] : Inflation Targeting at 20 : Achievements and Challenges, IMF working paper 09/236, International Monetary Fund.

S. Roger et M. Stone [2005] : On Target? The International Experience with achieving Inflation Targets, IMF working paper 05/163, International Monetary Fund.

T. J. Sargent [1999], The Conquest of American Inflation, Princeton: Princeton Univ. Press.

L. Svensson [2010] :Inflation Targeting, in B. Friedman et M. Woodford [eds.], Handbook of Monetary Economics, p. 1237-1302.

J.B. Van Huyck, Battalio J.C. et Walters M.F. [1995] : Commitment versus Discretion in the Peasant Dictator Game, Games and Economic Behavior, vol. 10, p. 143-170.

R. Vranceanu, D. Besancenot et D. Dubart [2013] : Can Rumors and Other Uninformative Messages Cause Illiquidity?, Essec Research Center WP, DR-130, Jul 13.

L. B. Willard [2012] :_Does inflation targeting matter? A reassessment, Applied Economics, vol. 44, $\mathrm{n}^{\circ} 17$, p. 2231-2244.

B. Wilson [1998] : Menu costs and nominal price friction: an experimental examination, Journal of Economic Behavior and Organization, vol. 35, p. 371-388.

M. Woodford [2003] : Interest and Prices: Foundations of a Theory of Monetary Policy, Princeton University Press.

\footnotetext{
${ }^{1}$ Voir Duffy [1998] puis [2008a] et également Ricciuti [2008].

2 Nous nous appuyons largement sur Cornand et Heinemann [2014b] qui proposent une revue de la littérature appliquant la macroéconomie expérimentale à la thématique des banques centrales hors champ de la politique monétaire.

${ }^{3}$ L'économie expérimentale est déjà utilisée comme outil de décision politique pour la conception de marchés dans des secteurs régulés (par exemple eau et électricité) et pour comparer des règles d'enchères (par exemple la conception d'enchères pour l'allocation des licences de téléphone mobile entre entreprises), pour la taxation, les biens publics, etc. Voir Ricciuti [2008].
} 
${ }^{4}$ Un argument communément avancé contre l'utilisation d'expériences de laboratoire comme outil d'aide à la décision politique est son manque supposé de validité externe : si les régularités observées en laboratoire ne sont pas vérifiées par des expériences de terrain (field experiments), les conclusions de politique pourraient être trompeuses [Riedl, 2010, p. 87]. Une façon de vérifier la validité externe est de remplacer les sujets qui sont généralement des étudiants par des sujets expérimentés et accoutumés à prendre le type de décisions à tester. Généralement, ce type d'expériences montre que les experts ne prennent pas de décisions significativement différentes de celles prises par des étudiants placés dans la même situation, bien que dans un certain nombre de cas il puisse y avoir des différences subtiles et surprenantes. Les expériences de terrain peuvent être considérées comme une méthode complémentaire. "Dans l'idéal, une proposition de politique économique devrait être évaluée à l'aune de toutes les méthodes scientifiques possibles : théoriquement, expérimentalement dans le laboratoire et sur le terrain et avec les méthodes économétriques traditionnelles » [Riedl, 2010, p. 88] («In the ideal case, an economic policy reform is evaluated with all possible scientific methods before a political decision is made. That is, theoretically, experimentally in the lab-and the field, and with traditional applied econometrics. »). Appliquer des expériences de terrain dans le domaine de la politique monétaire paraît difficilement envisageable dans la mesure où une banque centrale ne peut pas mettre en œuvre sa politique dans une zone restreinte de l'économie.

${ }^{5}$ Il a été Vice Chairman du Board of Governors du Federal Reserve System de juin 1994 à janvier 1996 et a écrit des papiers académiques utilisant des méthodes expérimentales (voir par exemple Blinder et Morgan [2005 et 2008]).

${ }^{6}$ La plupart des papiers présentés dans cet article s'appuient sur des expériences contextualisées dans lesquelles les sujets sont confrontés à des variables comme le niveau de l'emploi, des salaires, ou de l'inflation, plutôt qu'à une formulation abstraite (comme les règles de l'économie expérimentale l'exigent habituellement). L'explicitation du contexte peut être responsable de biais dans les comportements observés car les sujets peuvent être affectés par des jugements de valeur ou par leur propre expérience de la vie économique (en particulier lorsqu'on leur demande de former des anticipations). Pour éviter ces problèmes, il s'agirait de concevoir des expériences présentant un cadre abstrait, neutre (ou de faire suffisamment varier les paramètres des modèles pour obtenir des résultats robustes au cadre d'analyse). Ceci reste un défi pour la macroexpérimentation. De rares exceptions sont mentionnées plus bas.

${ }^{7}$ Un autre courant de la littérature suggère que la difficulté à rendre compte des phénomènes de persistance est le résultat d'une trop grande simplification des modèles conduisant à un mécanisme de propagation trop faible : des frictions plus importantes devraient être ajoutées aux modèles standards (voir par exemple Christiano et al. [2005]).

${ }^{8}$ L'illusion monétaire est définie comme la violation du postulat d'homogénéité (les fonctions d'offre et de demande sont homogènes de degré zéro dans tous les prix nominaux, de sorte qu'ils ne dépendent que des prix relatifs et non des prix absolus).

${ }^{9}$ Il existe également des expériences sur d'autres hypothèses à l'origine de la non neutralité monétaire comme les menus costs ou l'inattention rationnelle (rational inattention). Wilson [1998] considère des sujets en situation de monopole qui ajustent leur prix suite à des chocs et met en évidence le rôle des menus costs dans le processus d'ajustement des prix. Cheremukhin et al. [2011] testent la théorie de l'inattention rationnelle [Maćkowiak et Wiederholt, 2009], et montrent que l'information rigide peut être expliquée par l'inattention rationnelle.

${ }^{10}$ Kydland et Prescott sont les premiers à avoir mis en évidence l'incohérence temporelle liée à la politique monétaire (voir notamment Kydland et Prescott [1979]).

${ }^{11}$ Van Huyck, Battalio et Walters [1995] ont déjà proposé un test de l'incohérence temporelle liée à la mise en œuvre d'une politique discrétionnaire qui caractérise le modèle de Barro et Gordon, en recourant au jeu du paysan/dictateur : à chaque période, un paysan décide combien de haricots planter et donc du niveau de sa production. Dans le cas discrétionnaire, le dictateur décide du niveau de taxation de la production une fois que le paysan a décidé son niveau de production. Au contraire, dans le cas de l'engagement, à chaque période, le dictateur s'engage sur le taux de taxation avant que le paysan décide son niveau de production. Théoriquement, l'engagement permet d'assurer le niveau efficient de production. Ils implémentent ce jeu en laboratoire en considérant deux traitements: le premier représente le cas de la politique discrétionnaire et le second l'engagement. Le résultat principal de cette étude expérimentale est de montrer que la réputation ne peut pas se substituer à l'engagement.

${ }^{12}$ Les sujets qui anticipent l'inflation sont rémunérés selon une fonction de perte quadratique mesurant les déviations entre les anticipations et l'inflation effectivement réalisée. Les sujets jouant le rôle d'un banquier central sont rémunérés selon une fonction de perte avec deux termes quadratiques dépendant de la distance entre l'inflation et le chômage par rapport à leur niveau ciblé respectif. Selon la courbe de Phillips, ils doivent arbitrer entre le chômage et l'inflation. Ils ont à leur disposition un instrument, l'offre de monnaie, pour choisir entre différentes combinaisons possibles d'inflation et de chômage.

${ }^{13}$ L'économie est composée de sujets représentant les agents économiques qui font des prévisions d'inflation à chaque période de l'expérience. De plus, un sujet joue le rôle du décideur politique et fixe une cible d'inflation à chaque période. Ce dernier n'est pas en mesure de contrôler totalement le taux d'inflation. Le but principal d'Arifovic et Sargent est d'explorer si les sujets peuvent éviter l'équilibre de biais inflationniste du jeu à une période. Ils montrent qu'une majorité de leurs 12 groupes parvient le plus souvent à des taux d'inflation proches du taux zéro efficient (l'équilibre du jeu à une période n'est atteint que dans $5 \%$ des cas), mais dans quasiment tous les groupes, ils ont pu observer un nombre considérable de périodes pendant lesquelles l'inflation était élevée. Ceci peut être expliqué par le fait que les anticipations sont adaptatives. Les sujets jouant le rôle de banquiers centraux qui ont tenté de faire baisser les anticipations d'inflation n'ont pas suffisamment réduit les taux d'inflation ciblés par rapport à ce qui était nécessaire en considérant les meilleures réponses aux anticipations adaptatives.

${ }^{14}$ Selon Geraats [2002], la transparence politique est relative aux définitions et priorités affichées dans les objectifs, tandis que la transparence économique est relative aux informations économiques utilisées par la banque centrale.

${ }^{15}$ Voir Hommes [2011] pour une revue de la littérature. 
${ }^{16}$ Dans une économie expérimentale simple avec rigidité des prix, Adam [2007a] montre que les déviations des anticipations par rapport aux anticipations rationnelles contribuent à la persistance du produit et de l'inflation dans les modèles nouveaux keynésiens. Grâce aux données expérimentales qu'il obtient, il construit un modèle d'équilibre à perception restreinte (Restricted Perception Equilibrium model) et analyse les tendances cycliques de l'inflation autour de son état stationnaire.

${ }^{17}$ Dans la même direction, des niveaux limités de croyances d'ordre supérieur sur le fait que les autres prennent en compte le choc peuvent aussi expliquer de tels délais d'ajustement dans des jeux avec complémentarités stratégiques. Si le traitement de l'information est coûteux, il peut même être rationnel d'utiliser des degrés limités de croyances d'ordre supérieur.

${ }^{18}$ Leur but est exploratoire : il s'agit d'étudier si un certain nombre de faits stylisés connus peuvent être répliqués en laboratoire, d'évaluer dans quelle mesure les frictions jouent un rôle dans la réplication de ces faits stylisés et d'analyser la persistance des chocs dans de tels environnements. Les sujets prennent ainsi le rôle de consommateurs/travailleurs, producteurs, ou banquiers centraux. Un des principaux problèmes liés à l'implémentation de ces modèles macroéconomiques en laboratoire est qu'il est très difficile de capturer et d'analyser les croyances.

${ }^{19}$ La façon dont sont prises les décisions au sein de groupes varie assez largement; il y a généralement un leader, mais son autorité est variable. Par exemple, le Federal Open Market Committee sous Alan Greenspan était extrême, qualifié par Blinder et Wyplosz [2005] d'autocratique-collégial. A l'opposé, le comité de politique monétaire de la Banque d'Angleterre est qualifié de comité individuel par Blinder et le Conseil des Gouverneurs de la BCE de véritablement collégial. Pour davantage de détails sur la composition des comités de politique monétaire des économies ayant adopté des régimes de ciblage de l'inflation, voir Hammond [2012].

${ }^{20}$ « in almost all cases, once three or four subjects agreed on a course of action, the remaining one or two fell in line immediately. »

${ }^{21}$ La transparence partielle est plus simple à mettre en œuvre que la publicité partielle dans la mesure où les medias relayent l'information très rapidement à une large échelle. De plus, la publicité partielle pose des problèmes d'équité : il semble politiquement intenable pour une banque centrale de faire de la rétention d'information de manière intentionnelle dans une société démocratique.

${ }^{22}$ Une part importante de la littérature met en évidence le fait que les régimes explicites de ciblage de l'inflation ont généralement de bonnes performances macroéconomiques [Levin et al., 2004; Roger et Stone, 2005; Roger, 2009]. Au contraire, certaines études, parmi lesquelles celles de Ball et Sheridan [2005], Lin et Ye [2007], Angeriz et Arestis [2008], et Willard [2012] montrent que les bonnes performances macroéconomiques ne sont pas dues au ciblage d'inflation dans les pays de l'OCDE : les économies qui ont ciblé l'inflation, mais également celles qui ne l'ont pas fait, sont parvenues à maintenir des niveaux d'inflation faibles, ce qui suggère qu'une banque centrale n'a pas nécessairement besoin de mettre en place un régime de ciblage de l'inflation pour réaliser de bonnes performances macroéconomiques.

${ }^{23}$ Etant donnés les paramètres du modèle, le programme calcule les valeurs courantes des principales variables macroéconomiques.

${ }^{24}$ Le jeu de l'attaque spéculative présente des équilibres multiples si les fondamentaux de l'économie sont de connaissance commune parmi les spéculateurs. Il présente un équilibre unique si les agents ont de l'information privée suffisamment précise (comparativement à l'information publique qu'ils reçoivent sur les fondamentaux).

${ }^{25}$ Plus récemment, Morris et Shin [2014] présentent un modèle dans la même veine avec canal de la prise de risque de la politique monétaire.

${ }^{26}$ Plus précisément, Heinemann, Nagel et Ockenfels [2009] montrent que les comportements observés peuvent être décrits par un jeu global dans lequel les sujets se comportent comme s'ils obtenaient des signaux privés à propos des paiements. La technique de sélection d'équilibre proposée par les jeux globaux peut être utilisée pour rendre compte du comportement des agents sur les marchés financiers.

${ }^{27}$ Leurs résultats sont en ligne avec les autres études expérimentales sur les jeux de coordination: les sujets choisissent davantage l'option risquée lorsqu'ils ont plus d'information sur les gains potentiels liés à une telle option. Dans la même veine, un certain nombre d'études expérimentales montre que les sujets peuvent conditionner leurs actions à des signaux extrinsèques non pertinents ou taches solaires (sunspots) (voir par exemple Arifovic et Jiang [2013] pour une expérience sur un jeu de panique bancaire, Fehr et al. [2013] pour une expérience avec pur jeu de coordination, ou encore Marimon et al. [1993] et Duffy et Fisher [2005]). Vranceanu et al. [2013] mettent en évidence expérimentalement le fait que de fausses informations, des rumeurs et autres messages non informatifs peuvent créer de l'illiquidité dans un jeu d'investissement inspiré du modèle de panique bancaire de Diamond et Dybvig [1983]. 